\title{
Undergraduate dental education: an education or training?
}

\author{
Heidi Bateman, ${ }^{* 1}$ Jane Stewart, ${ }^{2}$ Giles McCracken ${ }^{3}$ and Janice Ellis ${ }^{4}$
}

\section{Key points}

Education and training have different purposes; both are important considerations when developing a dental graduate.
The different purposes of education and training are exemplified by the competing priorities between different stakeholders.
Acknowledging different agendas of those involved is the first stage in progressing towards a shared understanding of what the new graduate should 'be'.

\begin{abstract}
The impact of the COVID-19 pandemic on undergraduate dental education has led to new ways of working, different modes of delivery for elements of programmes and, in some cases, revised timelines for progression. These expedient changes, like the virus, may be with us for some time. However, as we come out of an intense period of critical reflection and change, it may also offer us an opportunity and impetus to revisit unresolved challenges around the focus and role of undergraduate dental education. We reflect upon the necessity for both clinical development and scholarship for our undergraduates, and the subtle differences of purpose between education and training. We consider whether it is time to look afresh at the relationship between the universities and other stakeholders and perhaps also to start with renewed understanding, giving deeper consideration to the value of each. One of the biggest obstacles to resolving these issues is to consider how best we align institutional requirements with the education and training of healthcare practitioners. With the hindsight of the past 12 months, we would argue that such a review is timely and more meaningful as we can reflect on the pandemic-enforced and expedient changes to our programmes and more clearly understand where our future priorities lie.
\end{abstract}

\section{Undergraduate dental education: an education or training?}

COVID-19 has created many changes to our personal and professional lives. Within undergraduate dental education, restrictions have meant reviewing the essential and not so essential elements of our curricula and in some instances has resulted in years of tradition being put aside and replaced by pragmatic decision-making. The custom and practice of programmes, often developmentally

${ }^{1}$ Clinical Trainer in Restorative Dentistry and Honorary Clinical Senior Lecturer, School of Dental Sciences, Newcastle University, Newcastle, UK; ${ }^{2}$ Senior Lecturer in Clinical Education, School of Medical Education, Newcastle University, Newcastle, UK; ${ }^{3}$ Professor of Restorative Dentistry and Honorary Consultant in Restorative Dentistry, School of Dental Sciences, Newcastle University, Newcastle, UK; ${ }^{4}$ Professor of Dental Education and Honorary Consultant in Restorative Dentistry, School of Dental Sciences, Newcastle University, Newcastle, UK. ${ }^{*}$ Correspondence to: Heidi Bateman

Email address: heidi.bateman@newcastle.ac.uk

Refereed Paper.

Accepted 19 July 2021

https://doi.org/10.1038/s41415-021-3653-0 constrained by legislation and regulation has, for this brief moment in time, refocused on the absolutely essential elements of professional educational practice. In doing so, new ways of working have been introduced and programme providers have had an opportunity to think anew about how best to develop our clinical practitioners. The hope by many of us is that this is only the beginning of positive changes and this period, although difficult, has created the conditions where we see the benefit of focusing on more searching questions, not just 'what we do' and 'how we do it' but 'why do we do it?' The following piece reflects our discussions on the, at times challenging, relationship between universities and other stakeholders in the development of new dental practitioners.

There has been a significant shift in the way new practitioners are inculcated into healthcare professions. From a historical apprenticeship-style model, characterised by imbibing knowledge with the formation of one's professional identity within the workplace, to a model of development that is embedded in university programmes and where increased institutional accountability is coupled with the overt labelling of professional standards. Dentistry is similar in these respects, where programmes are highly regulated and legislated at a national level through the governing body. The next evolutionary step in dentistry may be the introduction of new apprenticeships - espousing all of the advantages of the old system with the regulatory control of the current system.

The shift from the old apprenticeship training to university-based education can create challenges of competing agendas across two institutions: structured education within an academic setting, alongside immersion into a professional training experience. For programmes where graduation not only leads to an academic qualification but also allows registration with a professional regulator, we would argue that the incompatibility of different ways of viewing practitioner development remains unresolved, highlighting the differing priorities and demands of the various stakeholders invested 
in the output of healthcare graduates. These agendas are fuelled by a lack of clarity with regards to:

- The scholarship and the craft of the profession being differently valued by differing stakeholder groups

- The role the universities can and should play in training healthcare professionals

- The role of the workplace in the education and personal development of postgraduate trainees and going beyond their responsibility for training

- Whether the development of undergraduate and postgraduate personnel can and should be clearly delineated into 'education' and 'training' and the most suitable location for these activities is within universities and the workplace, respectively.

Although a significant overlap, it is accepted that education and training serve subtly different purposes within healthcare. ${ }^{1,2}$ The markers of successful education are concerned with preparing people to continue to function in a world beyond the here and now. Higher education facilitates and develops the more abstract values of wanting to learn, pursuing understanding and synthesising complex phenomenon. By doing so, the graduate will arguably be able to maintain their professional knowledge and skills by knowing how to act and be driven to do so by the need to adapt to future practices. For these reasons, education and the university experience prepare graduates for a broader and bigger world than just 'a job'. Training, in contrast, is more focused on equipping individuals for current practice, often characterised by standardised procedures, protocols and meeting defined competences. While we may assume that education is the remit of the universities and training within the workplace, it is important to remember that it is within the clinical context that contextualised, situational and complex knowledge is created and where the generic information of codified knowledge becomes the personal knowledge and skill set we require to function efficiently and effectively as a practitioner.

Challenges exist for education providers of dental programmes (within training) when they need to 'meet' wider university requirements and comply with their systems and protocols. University assessment frameworks prioritise elements such as the validity, reliability, fairness and defensibility of assessment, while in clinical contexts these frameworks are challenged by patient individuality and their variability. Clinical skills assessments have been developed for such occasions so that both sets of values can be, in theory, met. However, the necessity of removing the authenticity, contextual problems and 'realworld' complexity, to ensure each student has a similar experience and opportunity, results in a series of reductionist activities which become the measure of 'success'. In addition to the challenges this brings, there are also other highly desirable, nay, essential attributes which assessment frameworks cannot adequately capture in terms of their richness, complexity and responsiveness to context. ${ }^{3,4}$ There is no 'simple' solution to these challenges but they are worth acknowledging and discussing.

With its massive impact on workplace-based training and clinical experience, the pandemic has caused us to revisit questions of what are the 'essential elements' in undergraduate healthcare education and the competing dilemmas influencing these. As educators we have been forced to move rapidly to a largely online format and in doing so have lost elements of teaching normally delivered in person. But, perhaps in the case of many of these elements, the reason we were doing them in a face-to-face format was based on tradition and was simply the way things had always been done. For some elements moved to an online format, we would suggest the mode of delivery was less important than the content. In fact, the different delivery formats appeared to be appropriate and positively received and in some cases, possibly better received by current learners in preference to the traditional methods that have now been replaced. There is, however, anxiety as to how much is too much of a shift. For example, some programmes have seen a significant reduction in opportunities to gain operative and/or clinical experience, while others have seen clinical placements significantly altered to account for the rapidly changing operational conditions of placement providers.

In managing the pandemic, universities have focused on scholarship and recognise the impact of shifting educational delivery into a largely online format and how this effects programme quality assurance, academic standards and rigour, student engagement, attainment, wellbeing and satisfaction. Unsurprisingly, the General Dental Council as the regulatory body is focused on understanding the impact on clinical placements and the potentially reduced or altered clinical training experience. That is not to say that each organisation does not attach significance to the priorities of the other but they may value or evaluate elements in differing ways, depending on the principal function of each organisation. Education providers of dental programmes are concerned about both academic standards and clinical experience and therefore have had some challenging discussions and decisions to make as a result.

The mismatch of agenda has arisen within clinical education because we have worked within systems that have evolved over time and while not ideal, has largely worked by accommodating change. That is, up until now. The significant impact of the pandemic on all our educational and training processes has brought their weaknesses into stark relief and impacted far more on students' development than anyone would wish.

The first step towards managing competing agendas and 'tension' must surely be to acknowledge that they exist. The pandemic has shown where weaknesses are within our education and training and where unhelpful divides create challenges. Over the last year, there have been enormous steps forward in technologically-enhanced learning with innovative curricula solutions and developments. The time may now be right to discuss ideological values on the development of the 'professional' and return to first principles on what stakeholders each hope to achieve individually and collectively. This would complement the current debate on 'preparedness' of the new graduate, ${ }^{5,6,7,8,9,10}$ concerns of 'experience' and questions over the role of post-graduation schemes, such as Dental Foundation Training. Unless there is acknowledgement of tensions and competing agendas and consideration/agreement by stakeholders on what attributes the 'end product' (new graduate) can or should have, there will always be this mismatch of expectation and continued tensions. Repeated calls that graduates need different attributes and are not as they once were must surely be unsettling for our new graduates to hear, when they have met all the requirements set for them?

Without the ability to question, explore, understand and synthesise what constitutes a healthcare professional for a post-pandemic, twenty-first century practitioner, we as educators, curriculum designers, regulators etc are all unlikely to have the conceptual clarity to swiftly respond and adapt to the new ways of working demanded from us. 
What is clearer than ever is that 'future proofing' of our healthcare workforce is absolutely essential, with the wider skill set of the scholar and clinician being profoundly important and embedded within a university education. However, there is also a need for fora outside and across these institutions that allow difficult questions to be raised and discussed. Here, we could review the tenets that guide our decision-making and how to optimise a system reliant upon a synergy between the dual processes of education and training.

\section{Ethics declaration}

The authors declare no conflicts of interest.
Author contributions

All authors contributed to the discussions surrounding preparation and critical review of this article.

\section{References}

1. Key Differences. Difference Between Training and Education. Available at https://keydifferences.com/ difference-between-training-and-education.html (accessed May 2021).

2. HuffPost. Teach a Man to Fish: Training vs. Education. Available at https://www.huffpost.com/entry/teacha-man-to-fish-training-vs-education_b_7553264 (accessed May 2021).

3. Bateman H, Ellis J, McCracken G. Professionalism in undergraduate dental education: a pause for thought. BrDent J 2019; 227: 1025-1027.

4. Bateman H L, McCracken G I, Ellis J S, Stewart J. Delivering to 'that list': the challenges of working with learning outcomes. Br Dent J 2019; 226: 441-446.
5. Hanks S, Coelho C, Coster R. Prepared for practice and equipped for employment: what do dental foundation trainers think of their trainees? Br Dent J 2018; 225: 549-555.

6. Gilmour A S M, Jones R J, Cowpe J G, Bullock A D. Communication and professionalism skills of a new. graduate: the expectations and experiences of dental foundation trainers. Eur J Dent Educ 2014; 18: 195-202.

7. Gilmour A S M, Jones R J, Cowpe J G, Bullock A D. Clinical skills of a new foundation dentist: the expectations of dental foundation education supervisors. Br Dent J 2018; 225: 73-80.

8. Ali K, Tredwin C, Kay E J, Slade A, Pooler J. Preparedness of dental graduates for foundation training: a qualitative study. Br Dent J 2014; 217: 145-149.

9. Ali K, Slade A, Kay E, Zahra D, Tredwin C. Preparedness of undergraduate dental students in the United Kingdom: a national study. Br Dent J 2017; 222: 472-477.

10. General Dental Council. Preparedness for Practice of UK Graduates Report 2020. 2020. Available at https://www. gdc-uk.org/docs/default-source/research/preparednessfor-practice.pdf (accessed November 2021). 